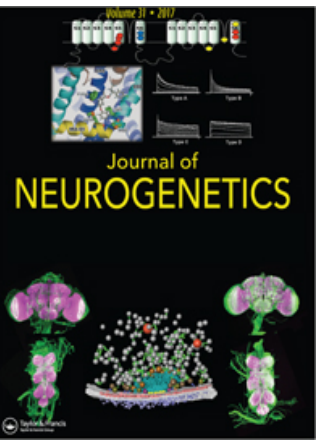

Journal of Neurogenetics

\title{
Drift diffusion model of reward and punishment learning in rare alpha-synuclein gene carriers
}

\author{
Ahmed A. Moustafa, Szabolcs Kéri, Bertalan Polner \& Corey White
}

To cite this article: Ahmed A. Moustafa, Szabolcs Kéri, Bertalan Polner \& Corey White (2017) Drift diffusion model of reward and punishment learning in rare alpha-synuclein gene carriers, Journal of Neurogenetics, 31:1-2, 17-22, DOI: 10.1080/01677063.2017.1301939

To link to this article: https://doi.org/10.1080/01677063.2017.1301939

\section{曲 Published online: 20 Mar 2017.}

Submit your article to this journal $\pi$

Џlll Article views: 102

View Crossmark data ¿ 


\title{
Drift diffusion model of reward and punishment learning in rare alpha-synuclein gene carriers
}

\author{
Ahmed A. Moustafa ${ }^{a}$, Szabolcs Kérib ${ }^{b, c, d}$, Bertalan Polner ${ }^{b, d}$ and Corey White ${ }^{e}$

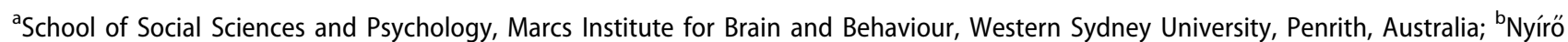 \\ Gyula Hospital, National Institute of Psychiatry and Addictions, Budapest, Hungary; ${ }^{\mathrm{C}}$ Faculty of Medicine, Department of Physiology, \\ University of Szeged, Szeged, Hungary; ${ }^{\mathrm{d}}$ Department of Cognitive Science, Budapest University of Technology and Economics, Budapest, \\ Hungary; ${ }^{e}$ Department of Psychology, Syracuse University, Syracuse, NY, USA
}

\section{ABSTRACT}

To understand the cognitive effects of alpha-synuclein polymorphism, we employed a drift diffusion model (DDM) to analyze reward- and punishment-guided probabilistic learning task data of participants with the rare alpha-synuclein gene duplication and age- and education-matched controls. Overall, the DDM analysis showed that, relative to controls, asymptomatic alpha-synuclein gene duplication carriers had significantly increased learning from negative feedback, while they tended to show impaired learning from positive feedback. No significant differences were found in response caution, response bias, or motor/encoding time. We here discuss the implications of these computational findings to the understanding of the neural mechanism of alpha-synuclein gene duplication.

\section{ARTICLE HISTORY}

Received 3 May 2016

Revised 23 February 2017

Accepted 28 February 2017

\section{KEYWORDS}

Alpha-synuclein gene; reinforcement learning; reward; punishment; Parkinson's disease; drift diffusion model

\section{Introduction}

The protein alpha-synuclein is the main component of Lewy-bodies, which are histological markers of neurodegeneration in Parkinson's disease (Goedert, Spillantini, Del Tredici, \& Braak, 2013). The rare duplications and triplications of the alpha-synuclein gene have repeatedly been shown to confer vulnerability to developing Parkinson's disease (Ahn et al., 2008; Elia et al., 2013; Ibanez et al., 2004; Nishioka et al., 2006), while large genome wide association studies have documented that common single nucleotide polymorphisms of alpha-synuclein are associated with risk of developing sporadic Parkinson's disease (Venda, Cragg, Buchman, \& Wade-Martins, 2010). Higher expression of alpha-synuclein has been associated with more severe phenotypes of familial Parkinson's disease (see Eriksen, Przedborski, \& Petrucelli, 2005). On the other hand, one study has found that the levels of alpha-synuclein in the cerebrospinal fluid were inversely correlated with the severity of motor abnormalities in patients with sporadic Parkinson's disease, as measured using the Hohn and Yahr scale (Tokuda et al., 2006).

Several lines of evidence have suggested that alphasynuclein could regulate dopaminergic neurotransmission at multiple stages (reviewed in Venda et al., 2010), so alphasynuclein duplications can be expected to influence learning from reward and punishment (Schultz, 2013). Analyzing optimal choices in a probabilistic classification task revealed the selective impairment of learning from reward in asymptomatic alpha-synuclein gene duplication carriers, whereas learning from punishment was intact (Keri, Moustafa, Myers, Benedek, \& Gluck, 2010). In the current study, we are applying drift diffusion models (DDM) to the dataset of the above study. Before introducing DDM, we will overview the scarce literature on human neurocognition in relation to the genetic regulation of alpha-synuclein.

Examining the same participants with alpha-synuclein gene duplication, another study reported normal delay discounting and caudate volume at asymptomatic stage (Szamosi, Nagy, \& Keri, 2013). Delay discounting is considered to be an indicator of impulsive decision making, and is measured with a task where participants choose between smaller, immediate, and larger, delayed rewards. At a later follow-up assessment, by the time all carriers have been diagnosed with Parkinson's disease, reduced caudate volumes and elevated delay discounting were found in the carriers. In two hundred healthy participants, Keri et al. (2008) examined haplotypes of the alpha-synuclein polymorphism that are known either to increase or decrease the risk of Parkinson's disease (Mueller et al., 2005). Participants with risk haplotypes were impaired in learning from rewarding feedback in a sequenced learning task, relative to participants with protective haplotypes. Additionally, no significant differences emerged between these two groups in terms of executive functions or sensory-motor skill learning.

To understand the cognitive effects of alpha-synuclein polymorphism, here we employed DDM to analyze the 
behavioral data of participants with alpha-synuclein gene duplication and controls. DDM are class of models that analyze the relationship among accuracy and reaction times (Ratcliff \& McKoon, 2008). DDM assume that decisions involve the gradual accumulation of noisy evidence until a criterial amount is reached. In the model, the decision process starts between two boundaries that correspond to the response options. Over time, noisy evidence from a stimulus is sampled and accumulated until the process reaches a boundary, signaling the commitment to that response. The time taken to reach the boundary corresponds to the decision time, and the overall response time is given the decision time plus residual non-decision time. This model has been successfully used in the past to explain decision making (Krajbich, Lu, Camerer, \& Rangel, 2012; Petrov, Van Horn, \& Ratcliff, 2011; White, Ratcliff, Vasey, \& McKoon, 2010) as well as learning data (Moustafa, Keri, et al., 2015). As compared to the analysis of plain hit rates or reaction times, DDM provides valuable additional information: it simultaneously considers accuracy and response speed, thus allows separate examination of factors determining performance, such as speed-accuracy thresholds, response bias, and learning rate (Ratcliff, Smith, Brown, \& McKoon, 2016).

We have used DDM in the past to disentangle learning performance in patients with schizophrenia (Moustafa, Keri, et al., 2015). In the current study, we are applying DDM to neurogenetics and learning data (Keri et al., 2010). We focus on understanding the effects of the alpha-synuclein gene duplication on the computational mechanisms of reward and punishment learning.

\section{Methods}

\section{Participants}

We recruited seven Caucasian participants, who were siblings of three patients with Parkinson's disease with alpha-synuclein gene duplication. The siblings were asymptomatic carriers of alpha-synuclein gene duplication at the time of behavioral assessment. During a subsequent follow-up period, all carriers developed Parkinson's disease and a marked cognitive decline, as revealed by the Mini-Mental State Examination (Folstein, Folstein, \& McHugh, 1975) [baseline: $30.0(\mathrm{SD}=0)$, follow-up: $22.4(\mathrm{SD}=2.1)$ ]. The comparison group included 10 healthy volunteers without alpha-synuclein gene duplication. There was no familial relatedness among controls. The carriers and the controls were matched for age, gender, education, IQ, and Hollingshead's socioeconomic status (Table 1). All participants were screened with the Structured Clinical Interview for DSM-IV Axis I Disorders, Clinician Version (First, Gibbon, Spitzer, \& Williams, 1996)

Table 1. Demographic characteristics of the control and asymptotic alpha-synuclein gene duplication carrier participants.

\begin{tabular}{lcc}
\hline & Asymptotic carriers & Controls \\
\hline Age (years) & $47.7(8.6)$ & $45.6(8.2)$ \\
Males/females & $5 / 2$ & $7 / 3$ \\
Education (years) & $13.0(3.5)$ & $12.2(3.6)$ \\
IQ & $107.6(14.2)$ & $109.3(11.2)$ \\
Socioeconomic status & $37.6(5.3)$ & $36.7(5.6)$ \\
\hline
\end{tabular}

and underwent a detailed neurological examination including routine head MRI and [123I] $\beta$-CIT SPECT. These assessments revealed no psychiatric disorders, neurological signs and symptoms, neuropsychological deficits, and dopamine transporter abnormalities in the participants at the time of behavioral testing. All participants gave written informed consent, and the study was approved by the local ethics board. The present study is a reanalysis of behavioral data previously published (Keri et al., 2010). As the previous publication did not involve analysis of reaction times, here we present the simultaneous analysis of reaction times and accuracy using DDM.

\section{Task}

Participants performed a reward- and punishment-guided probabilistic learning task (Keri et al., 2010; Moustafa, Gluck, Herzallah, \& Myers, 2015; Moustafa, Sheynin, \& Myers, 2015; Myers et al., 2016). Briefly, they were instructed that they could win or lose imaginary quarter dollars by deciding whether abstract images belong to category A or B. Some images belonged to category A with $80 \%$ probability and to category B with $20 \%$ probability, while others belonged to category B with $80 \%$ probability and to category A with $20 \%$ probability. On reward-learning trials, participants received 25 points for correct decisions, whereas incorrect guesses were not followed by any feedback. On punishment-learning trials, incorrect decisions resulted in the loss of 25 points, whereas correct guesses received no feedback. Reward and punishment trials were intermixed so that no-feedback trials were potentially ambiguous.

\section{Statistical analysis}

In the DDM, non-decision time (Ter) accounts for the duration of processes outside the decision itself, namely encoding of the stimulus and execution of the motor response. In addition to the non-decision time component, DDM has three primary components that affect decisions. The distance between the two boundaries (a-0), gives indices of response caution or speed/accuracy settings. A wide boundary separation means that more evidence needs to be sampled to reach a boundary, so responses will be slower. But, at the same time, the decision process is less likely to reach the wrong boundary due to noisy evidence, so responses are simultaneously more accurate. Thus, boundary separation indicates how much evidence is required before committing to the response and provides a measure of the speed/accuracy tradeoff. The starting point of evidence accumulation $(z)$, indicates a response bias for one option over the other. If the starting point is closer to one boundary, less evidence is required to reach that decision than the alternative. Thus if the starting point is closer to boundary A, responses for Option A will be more probable and faster than for Option B. Finally, the drift rate $(v)$ gives an index of the direction and strength of the stimulus evidence driving the accumulation process. Positive values of drift rate indicate evidence for Option A and negative values indicate evidence for 
Option B. Further, a large absolute value of drift rate indicates very strong evidence for that option, which will result in fast responses and a high probability of choosing that option. The drift rate is tied to the task at hand, in this case it would indicate how well the participant has learned to correctly classify the stimuli after learning the reward and punishment contingencies.

A DDM was fitted to each participant's behavioral data using the $X^{2}$ method (Ratcliff \& Tuerlinckx, 2002). The 0.1, $0.3,0.5,0.7$, and 0.9 quantiles of the reaction time distribution were calculated for both correct and error responses to represent the shape of the distributions. These quantiles were entered into the fitting routine along with the choice probabilities. Then the fitting routine uses a simplex algorithm (Nelder \& Mead, 1965) to adjust the parameter values and find the ones that provide the closest match to the observed data (by minimizing the $X^{2}$ value). This process allows for the estimation of the different decision components in the DDM.

\section{Results}

The results of the DDM parameter comparisons are listed below. First, we ensured that the model fit the behavioral data well. Figure 1 shows the observed data plotted alongside the predicted data from the best fitting DDM parameters. The strong correspondence shows the model captured the data well.

Comparisons of the DDM parameters involved Wilcoxon-Mann-Whitney's tests between carriers and controls, with Monte-Carlo approximation for 100,000 random samples (Figure 2). Statistical analyses were performed with R (R Core Team, 2016, version 3.3.1), using the coin package (Zeileis, Wiel, Hornik, \& Hothorn, 2008).

There were no differences between controls and carriers for comparison of motor/encoding duration (nondecision time; $Z=-0.68, p=.53$ ), response caution (boundary separation; $Z=1.17, p=.26$ ), or response bias (starting point;

\section{Response Proportions}

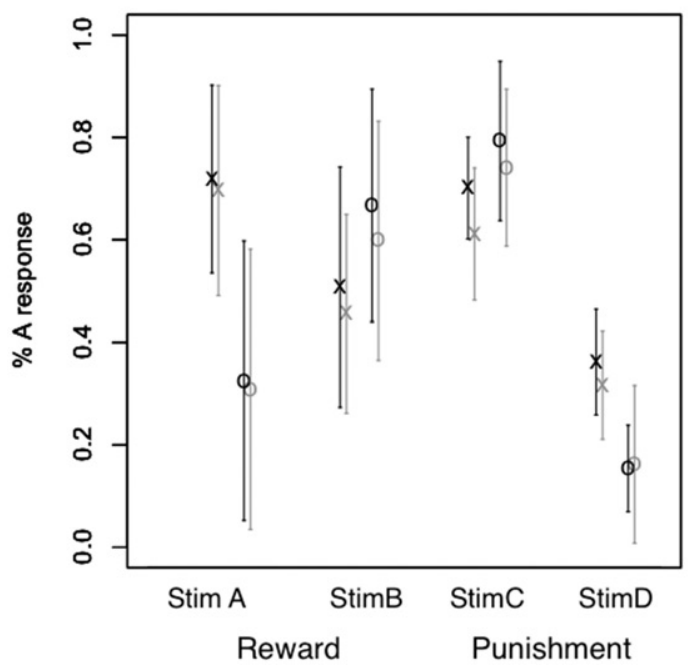

$Z=0.10, p=.96)$. Learning was assessed by comparing the drift rate discriminability measure, which was calculated as the difference in drift rates for left and right response stimuli. In Figure 2, higher discriminability values indicate better learning of the stimulus-response pairing, and negative values indicate reversed learning where the incorrect response is given more often than the correct response. Controls tended to show stronger discriminability than carriers for reward trials $(Z=1.95, p=.0548)$ and yet significantly poorer discriminability for punishment trials $(Z=-2.63$, $p=.007)$.

In addition, to compare learning from punishment and reward within both groups, we performed Wilcoxon's signed-rank tests with Monte-Carlo approximation for 100,000 random samples. Carriers demonstrated significantly better learning from punishment, as compared to learning from reward $(Z=2.37, p=.016)$, while no significant difference was found between learning from punishment vs. reward in controls $(Z=-0.15, p=.922)$.

\section{Discussion}

Overall, the DDM analysis showed that asymptomatic alphasynuclein gene duplication carriers significantly differed from controls in learning from negative feedback, while they demonstrated marginally impaired learning from positive feedback. Carriers demonstrated significantly better learning from negative feedback, relative to learning from positive feedback, while no such difference was found among controls. Going beyond our previous study (Keri et al., 2010), here we described the altered computations underlying the reinforcement learning deficits of asymptomatic alphasynuclein gene duplication carriers. Intriguingly, DDM have presented evidence for elevated processing of punishment in carriers, and tended to confirm the formerly reported reward learning impairment.

No significant differences were found in response caution, response bias, or motor/encoding time. Although the lack of

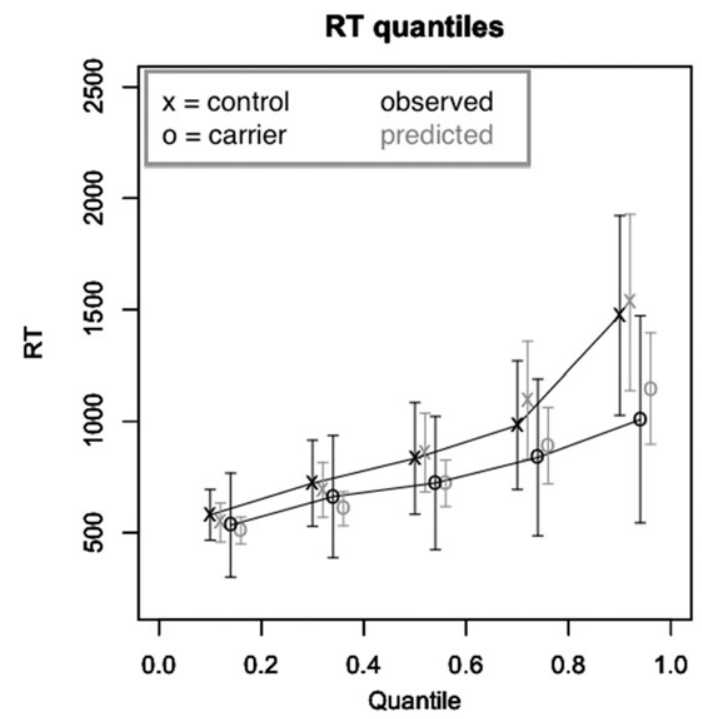

Figure 1. Observed vs. predicted data from the drift diffusion model (DDM). Error bars reflect $95 \%$ confidence intervals. Carriers refer to alpha synuclein gene duplication carriers. 

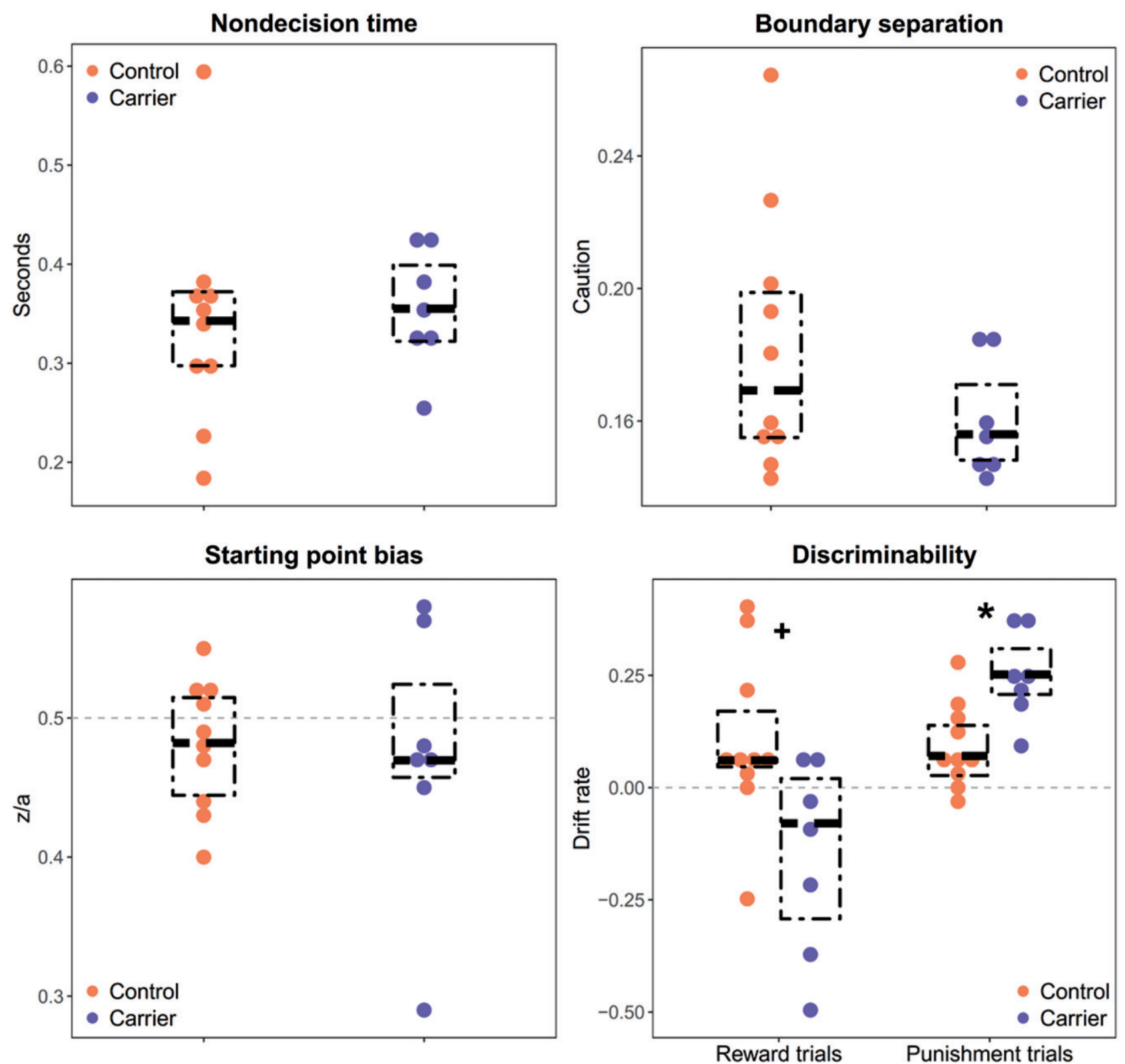

Figure 2. DDM parameters for subjects. The thick lines in the middle of the boxes indicate the medians, while the lower and the upper borders of the boxes indicate the 1 st and the 3 rd quartile, respectively. Carriers refer to alpha synuclein gene duplication carriers. ${ }^{*} p=.007 ;{ }^{+} p=.0548$.

significant differences should be interpreted with caution given the low sample size, we attempt to link these null findings to the related literature. First, in contrast to the previously reported association of alpha-synuclein levels in the cerebrospinal fluid and motor symptoms in patients with Parkinson's (Tokuda et al., 2006), alpha-synuclein gene duplication did not impact motor/encoding time. This is possibly related to minor or often non-existent motor dysfunction in individuals with alpha-synuclein gene duplications or triplications. Future work should apply DDM analysis to learning and decision making data from Parkinson's disease patients.

Additionally, no significant increase in impulsive decision making has previously been documented in the carriers at the asymptomatic stage (Szamosi et al., 2013). DDM suggested no significant differences in response caution, as compared to controls, which might parallel the former findings of normal delay discounting. Furthermore, in line with the present results, healthy carriers of the alpha-synuclein risk haplotype were deficient in learning from positive feedback, while they did not differ significantly from protective haplotype carriers in terms of executive functions and rudimentary sensory-motor skills (Keri et al., 2008). Along the same lines, we found no significant difference between carriers and controls in motor and encoding duration. Finally, the present results remarkably differ from those obtained in our prior DDM study in schizophrenia (Moustafa, Keri, et al., 2015). Relative to controls, patients with schizophrenia had prolonged motor/encoding time, responded more cautiously, and demonstrated deficient learning from punishment. In alpha-synuclein gene duplication carriers, the drift rate parameters showed an interesting pattern related to learning from feedback: compared to controls, carriers tended to show poorer learning for reward trials, that bordered on 
reverse learning (i.e. learned the incorrect response rather than the correct one), yet significantly stronger learning for punishment trials. Additionally, carriers learned better from negative feedback, as compared to learning from positive feedback. This pattern is similar to what previously has been reported for unmedicated patients with idiopathic Parkinson's disease (Bódi et al., 2009), suggesting that bias towards learning from negative feedback might be a general feature that characterizes both asymptomatic alpha-synuclein duplication carriers and unmedicated patients with Parkinson's disease.

Curiously, the present findings somewhat contrast with the selective reward learning deficit previously reported solely on the basis of accuracy data (Keri et al., 2010). The discrepancy might be due to the fact that DDM simultaneously takes accuracy and reaction time into account, and disentangles various factors influencing behavior, thereby increases statistical power for factors of interest (Ratcliff et al., 2016). Although no significant differences were found in terms of motor/encoding duration, response caution, or response bias between carriers and controls, controlling for their effect nevertheless revealed a significant deficit of learning from punishments in carriers. We see the application of a sophisticated analytical technique to behavioral data as a remarkable strength of the present study, as it provided novel clues about the mechanisms underlying cognitive deficit in alpha-synuclein gene duplication carriers. On the other hand, the low sample size is an important limitation, which might appear more acceptable in light of the rarity of alpha-synuclein gene duplication.

Alpha-synuclein gene duplication can cause disturbances in dopaminergic neurotransmission (Venda et al., 2010), which might be reflected in altered learning from reward and punishment. Beyond rare risk mutations and multiplications (Eriksen et al., 2005), common polymorphisms of alpha-synuclein are also associated with risk of sporadic Parkinson's disease (Venda et al., 2010). However, little is known about the influence of these polymorphisms on reward and punishment learning (Keri et al., 2008). Future research should investigate whether the altered pattern of learning from reward and punishment can be considered a behavioral indicator of genetic risk for sporadic Parkinson's disease.

\section{Disclosure statement}

The authors declare they have no conflict of interest.

\section{References}

Ahn, T.B., Kim, S.Y., Kim, J.Y., Park, S.S., Lee, D.S., Min, H.J., ... Jeon, B.S. (2008). alpha-Synuclein gene duplication is present in sporadic Parkinson disease. Neurology, 70, 43-49. doi:10.1212/ 01.wnl.0000271080.53272.c7

Bódi, N., Kéri, S., Nagy, H., Moustafa, A., Myers, C.E., Daw, N., ... Gluck, M.A. (2009). Reward-learning and the novelty-seeking personality: A between- and within-subjects study of the effects of dopamine agonists on young Parkinson's patients. Brain, 132, 2385-2395. doi: 10.1093/brain/awp094
Elia, A.E., Petrucci, S., Fasano, A., Guidi, M., Valbonesi, S., Bernardini, L., ... Valente, E.M. (2013). Alpha-synuclein gene duplication: Marked intrafamilial variability in two novel pedigrees. Movement Disorders, 28, 813-817. doi:10.1002/mds.25518

Eriksen, J.L., Przedborski, S., \& Petrucelli, L. (2005). Gene dosage and pathogenesis of Parkinson's disease. Trends in Molecular Medicine, 11, 91-96. doi:10.1016/j.molmed.2005.01.001

First, M.B., Gibbon, M., Spitzer, R.L., \& Williams, J.B.W. (1996) Structured clinical interview for DSM-IV Axis I disorders, clinician version. Washington, DC: American Psychiatric Press.

Folstein, M.F., Folstein, S.E., \& McHugh, P.R. (1975). "Mini-mental state": A practical method for grading the cognitive state of patients for the clinician. Journal of Psychiatric Research, 12, 189-198. doi:10.1016/0022-3956(75)90026-6

Goedert, M., Spillantini, M.G., Del Tredici, K., \& Braak, H. (2013). 100 years of Lewy pathology. Nature Reviews Neurology, 9, 13-24. doi:10.1038/nrneurol.2012.242

Ibanez, P., Bonnet, A.M., Debarges, B., Lohmann, E., Tison, F., Pollak, P., ... Brice, A. (2004). Causal relation between alpha-synuclein gene duplication and familial Parkinson's disease. Lancet, 364, 1169-1171. doi: 10.1016/S0140-6736(04)17104-3

Keri, S., Moustafa, A.A., Myers, C.E., Benedek, G., \& Gluck, M.A. (2010). \{alpha\}-Synuclein gene duplication impairs reward learning. Proceedings of the National Academy of Sciences of the United States of America, 107, 15992-15994. doi:10.1073/pnas.1006068107

Keri, S., Nagy, H., Myers, C.E., Benedek, G., Shohamy, D., \& Gluck, M.A. (2008). Risk and protective haplotypes of the alpha-synuclein gene associated with Parkinson's disease differentially affect cognitive sequence learning. Genes, Brain and Behavior, 7, 31-36. Retrieved from http://www.ncbi.nlm.nih.gov/entrez/query.fcgi?cmd=Retrieve $\& \mathrm{db}=$ PubMed\&dopt $=$ Citation\&list_uids $=17451452$

Krajbich, I., Lu, D., Camerer, C., \& Rangel, A. (2012). The attentional drift-diffusion model extends to simple purchasing decisions. Frontiers in Psychology, 3, 193. doi:10.3389/fpsyg.2012.00193

Moustafa, A.A., Gluck, M.A., Herzallah, M.M., \& Myers, C.E. (2015). The influence of trial order on learning from reward versus punishment in a probabilistic categorization task: Experimental and computational analyses. Frontiers in Behavioral Neuroscience, 9, 153.

Moustafa, A.A., Keri, S., Somlai, Z., Balsdon, T., Frydecka, D., Misiak, B., \& White, C. (2015). Drift diffusion model of reward and punishment learning in schizophrenia: Modeling and experimental data. Behavioural Brain Research, 291, 147-154. doi:10.1016/ j.bbr.2015.05.024

Moustafa, A.A., Sheynin, J., \& Myers, C.E. (2015). The role of informative and ambiguous feedback in avoidance behavior: Empirical and computational findings. PLoS One, 10, e0144083. doi:10.1371/ journal.pone. 0144083

Mueller, J.C., Fuchs, J., Hofer, A., Zimprich, A., Lichtner, P., Illig, T., ... Gasser, T. (2005). Multiple regions of alpha-synuclein are associated with Parkinson's disease. Annals of Neurology, 57, 535-541. doi:10.1002/ana.20438

Myers, C.E., Sheynin, J., Balsdon, T., Luzardo, A., Beck, K.D., Hogarth, L., .. Moustafa, A.A. (2016). Probabilistic reward- and punishment-based learning in opioid addiction: Experimental and computational data. Behavioural Brain Research, 296, 240-248. doi:10.1016/ j.bbr.2015.09.018

Nelder, J.A., \& Mead, R. (1965). A simplex method for function minimization-Nelder. The Computer Journal, 7, 308-313. doi:10.1093/ comjnl/7.4.308

Nishioka, K., Hayashi, S., Farrer, M.J., Singleton, A.B., Yoshino, H., Imai, H., ... Hattori, N. (2006). Clinical heterogeneity of alpha-synuclein gene duplication in Parkinson's disease. Annals of Neurology, 59, 298-309. doi:10.1002/ana.20753

Petrov, A.A., Van Horn, N.M., \& Ratcliff, R. (2011). Dissociable perceptual-learning mechanisms revealed by diffusion-model analysis Psychonomic Bulletin \& Review, 18, 490-497. doi:10.3758/s13423011-0079-8

$\mathrm{R}$ Core Team. (2016). R: A language and environment for statistical computing. Vienna, Austria: R Foundation for Statistical Computing. Retrieved from http://www.R-project.org 
Ratcliff, R., \& McKoon, G. (2008). The diffusion decision model: Theory and data for two-choice decision tasks. Neural Computation, 20, 873-922. doi:10.1162/neco.2008.12-06-420

Ratcliff, R., \& Tuerlinckx, F. (2002). Estimation of the parameters of the diffusion model: Approaches to dealing with contaminant reaction times and parameter variability. Psychonomic Bulletin and Review, 9, 438-481. doi:10.3758/BF03196302

Ratcliff, R., Smith, P.L., Brown, S.D., \& McKoon, G. (2016). Diffusion decision model: Current issues and history. Trends in Cognitive Sciences, 20, 260-281. doi: 10.1016/j.tics.2016.01.007

Schultz, W. (2013). Updating dopamine reward signals. Current Opinion in Neurobiology, 23, 229-238. doi:10.1016/j.conb.2012.11.012

Szamosi, A., Nagy, H., \& Keri, S. (2013). Delay discounting of reward and caudate nucleus volume in individuals with alphasynuclein gene duplication before and after the development of Parkinson's disease. Neurodegenerative Diseases, 11, 72-78. doi:10.1159/000341997
Tokuda, T., Salem, S.A., Allsop, D., Mizuno, T., Nakagawa, M., Qureshi, M.M., ... El-Agnaf, O.M. (2006). Decreased alpha-synuclein in cerebrospinal fluid of aged individuals and subjects with Parkinson's disease. Biochemical and Biophysical Research Communications, 349, 162-166. doi:10.1016/j.bbrc. 2006. 08.024

Venda, L.L., Cragg, S.J., Buchman, V.L., \& Wade-Martins, R. (2010). $\alpha$-Synuclein and dopamine at the crossroads of Parkinson's disease. Trends in Neurosciences, 33, 559-568. doi:10.1016/ j.tins.2010.09.004

White, C.N., Ratcliff, R., Vasey, M.W., \& McKoon, G. (2010). Anxiety enhances threat processing without competition among multiple inputs: A diffusion model analysis. Emotion, 10, 662-677. doi:10.1037/a0019474 2010-22093-006

Zeileis, A., Wiel, M.A., Hornik, K., \& Hothorn, T. (2008). Implementing a class of permutation tests: The coin package. Journal of Statistical Software, 28, 1-23. doi:10.18637/jss.v028.i08 